\title{
Acupuncture for relief of gag reflex in patients undergoing transesophageal echocardiography - a protocol for a randomized sham-controlled trial
}

Taras Usichenko ( $\square$ taras.usichenko@med.uni-greifswald.de )

Universitatsmedizin Greifswald https://orcid.org/0000-0002-6173-5436

Irina Mueller-Kozarez

Universitatsmedizin Greifswald

Stephan Knigge

Universitatsmedizin Greifswald

Klaus Hahnenkamp

Universitatsmedizin Greifswald

Raila Busch

Universitatsmedizin Greifswald

Mathias Busch

Universitatsmedizin Greifswald

Study protocol

Keywords: transesophageal echocardiography, gag reflex, acupuncture

Posted Date: September 17th, 2019

DOI: https://doi.org/10.21203/rs.2.14231/v1

License: () (1) This work is licensed under a Creative Commons Attribution 4.0 International License.

Read Full License 


\section{Abstract}

Background Gagging during transesophageal echocardiography examination (TEE) can be both distressing and even dangerous for patients. Needling of acupuncture point CV24 was described to be effective in reducing of gag reflex during TEE in patients with ischemic stroke or transient ischemic attack.

Methods/Design We describe a proposal for a prospective randomized, patient, practitioner and assessorblinded, single-center trial with two arms/groups: real acupuncture will be compared to placebo acupuncture. A total of 60 (30 per group) patients scheduled for elective TEE in order to exclude a cardiac embolic source, endocarditis or for valve failure evaluation will be recruited according to patients' selection criteria and receive either indwelling fixed intradermal needles at acupoints CV24 and bilateral PC6 or sham acupuncture at the same sites. Patients undergoing TEE, practitioners performing TEE, and the outcome assessors will be unaware of group (real or placebo) allocation. The primary outcome is the intensity of gagging, measured using verbal rating scale (VRS-11) from $0=$ no gagging to $10=$ intolerable gagging. Secondary outcomes include the incidence of gagging, the use of rescue medication, patients' satisfaction with relief of unwanted side effects during the TEE procedure, success of patients' blinding (patients' opinion to group allocation), heart rate and oxygen saturation measured by pulse oxymetry.

Discussion To study the effects of acupuncture to reduce gagging during TEE we test needling of the acupoints CV24 and PC6 bilaterally. Placebo acupuncture is used for the control group.

\section{Background}

Gag reflex is a part of a normal defense mechanism, intended to prevent unwanted, irritating, or toxic material or fluids from entering the trachea, pharynx or larynx. It is a polysynaptic reflex, which arises at the cortical level and is composed of a palatal and a pharyngeal response. The palatal response comprises upward movement of the soft palate with synchronous deviation of the uvula, whereas the pharyngeal response involves contraction of the pharyngeal wall $(1,2)$. Concomitant features include salivation, eye watering, coughing, sweating, head withdrawal, fainting or even a panic attack (3). The gag reflex can be stimulated by touching trigger areas such as the posterior pharyngeal wall, the tonsillar area, the base of the tongue, the palate and uvula.

With the growing number of diagnostic and therapeutic procedures, where manipulation in the pharyngeal area is necessary (e.g. with fiberoptic scopes), gagging has become a common clinical problem $(4,5)$. Gagging makes diagnostic and therapeutic procedures distressing and often difficult or even impossible to perform. Various interventions are used to control the gag reflex; for example, antiemetic medication, sedatives, local and general anesthetics, herbal remedies, behavioral therapies, acupressure, acupuncture, and prosthetic devices; however, there is no evidence favoring any specific treatment of gag reflex in clinical conditions (5). 
Needling of acupuncture points CV24 and PC6 (Figure 1), is suggested as a complementary or even alternative option for treatment of gagging and concomitant nausea during various medical procedures $(6-11)$.

Acupuncture of CV24 and PC6 is commonly used to alleviate the gag reflex in dentistry in adult and in pediatric patients. The effectiveness was demonstrated in several pilot case series and a few randomized controlled investigations $(6,7,10,11)$. Beyond dentistry, needle stimulation of the acupoint CV24 was shown to be effective for suppressing gag reflex in patients with ischemic stroke or transient ischemic attack undergoing transesophageal echocardiography (TEE) (9). In this investigation acupuncture was superior to a sham procedure, was easy to apply, and was effective in reducing the gag reflex during TEE.

We are going to develop an effective, easily applied method to relieve the gag reflex using needling of acupoints CV24 and PC6 in patients scheduled for ambulatory TEE for cardiologic diagnostics. For this purpose we will compare acupuncture of CV24 and PC6 with a sham procedure in a two-arm randomized controlled investigation, where the patients, cardiologists and outcome assessors will be blinded to group allocation.

\section{Objective}

The aim is to investigate whether CV24 and PC6 acupuncture can relieve gagging in cardiologic patients during ambulatory TEE and whether real acupuncture is superior to a sham procedure.

\section{Methods}

\section{Study design}

This investigation is a prospective randomized, patient, practitioner and assessor-blinded, single-center trial at the Medical University of Greifswald with two arms/groups: real acupuncture will be compared to non-penetrating sham acupuncture (Figure 1). The total duration of the investigation for each patient is approximately 30 minutes. A total of 60 (30 per group) patients scheduled for elective transesophageal echocardiography (TEE) will be recruited according to patients' selection criteria. TEE will be performed in preparation for an electrophysiological procedure, to exclude a cardiac embolic source or endocarditis or for valve failure evaluation. Patients undergoing TEE, practitioners performing TEE and outcome assessors will be unaware ("blinded") of the group allocation. Only the acupuncturist, who will perform randomization, will know the group allocation. Local ethics committee approved the protocol of the investigation and this study was registered as NCT0382142.

\section{Inclusion criteria}


- patients with an American Society of Anesthesiologists physical status of I to III scheduled for elective ambulatory transesophageal echocardiography (TEE) without sedation

- without previous opioid and psychotropic medication

- age between 19 and 65 years, able to use verbal rating scale $100 \mathrm{~mm}$

- patients who have given written informed consent to participate in the trial and to publish the data of the trial

\section{Exclusion criteria}

- skin infection at the sites of acupuncture

- patients who consume opioid or psychotropic medication

- patients with the history of: i) psychiatric disease; ii) radio- or chemotherapy; iii) peripheral polyneuropathy.

- patients who are unable to understand the consent form or to use verbal rating scale $100 \mathrm{~mm}$.

\section{Discontinuation criteria}

- in case of the necessity to change the usual TEE schedule for a particular patient

- complications during the TEE procedure (including inability to introduce the transesophageal probe)

- all patients are free to withdraw from participation in this study at any time, for any reason, specified or non-specified, and without penalty or loss of benefits to which a patient is otherwise entitled.

- Group allocation and randomization

During the standard pre-procedural examination, the patients, screened according to eligibility criteria (see above), will be informed about the possibility of receiving either real acupuncture (RA) or non-penetrating sham acupuncture (SA) by chance in addition to standard therapy for relief of the gag reflex during TEE and will be asked if they would like to take part in the investigation. If they agree, they will have to sign the informed consent and will be randomly allocated to RA (30 patients) or SA (30 patients) prior to TEE procedure (Figure 1 and Figure 2). The acupuncturist will perform the allocation procedure using a computer-generated list of random numbers. The randomization is concealed from patients and practitioners; only the acupuncturist will be aware of group allocation.

\section{Study procedure}

In RA group, the acupuncturist will apply the needles to Chengjiang CV24 and Neiguan PC6 (bilaterally) acupuncture points immediately prior to TEE procedure. PC6 is situated on the medial side of lower arm between the tendons of $\mathrm{M}$. flexor carpi radialis and $\mathrm{M}$. palmaris longus $3-4 \mathrm{~cm}$ proximal to the wrist 
crease (Figure 3). CV24 is situated on the ventral midline in the mento-labial groove (Figure 4). The choice of acupuncture points is based on the findings from previous investigations on the treatment of gagging in patients during medical procedures (6-11). For acupuncture at PC6, disposable indwelling New Pyonex acupuncture needles (diameter 0.2 and length $1.5 \mathrm{~mm}$, manufactured by Seirin Corp., Japan) embedded in skin-colored adhesive tape will be used (Figure 2B, 2C). Acupuncture at CV24 will be done using disposable intradermal "Spinex" needles (diameter $0.14 \mathrm{~mm}$ and length $6 \mathrm{~mm}$; Seirin Corp. Japan, Figure 4B). A Spinex needle will be inserted and its loop, which remains on the surface of the skin (Figure 4C), will be covered using a New Pyonex sham needle (Figure 4D). The New Pyonex sham needle, which was designed as a sham control in auricular acupuncture studies, has the same appearance as a New Pyonex needle but consists of self-adhesive tape and plastic bead only without the needle itself (Figure 4C).

In patients, who will be assigned to SA group, the acupuncturist will apply the New Pyonex sham needles to the same areas, where the needles for RA would be inserted. In order to provide the feeling of "pricking" during the application of New Pyonex sham needles, the skin areas around the acupuncture points will be examined using the SVESA neural pen (Neuralstift SVESA 1070, SVESA, Muenchen, Germany). The SVESA neural pen is commonly used in acupuncture practice for identification of skin areas with lower skin resistance, which are attributed to acupuncture points, as was described elsewhere (12). The neural pen has a thin tip, which produces the feeling of needle insertion, if certain pressure is applied to the skin. The acupuncturist will tell the patients from the SA group that he is using the neural pen "to find" the acupuncture points; however; he will use it to produce a pressure with the tip of neural pen (resembling the pricking of the needle) and attach New Pyonex sham needles at the areas of the skin near the acupuncture points but not at the acupoints exactly.

The acupuncture needles or sham needles will be placed immediately before the TEE procedure retained in situ during the diagnostic procedure and will be taken out after the TEE probe is withdrawn. Before the needle insertion, the skin will be wiped with alcohol swabs.

\section{Outcome Measures}

The practitioner, who will perform TEE, will also register the outcome measures immediately following the end of TEE procedure and withdrawal of the needles (Figure 2).

Primary outcome measure: intensity of gagging, reported by the patient using the verbal rating scale (VRS-11) from $0=$ no gagging at all to $10=$ maximally uncomfortable under gagging .

\section{Secondary outcome measures:}

- incidence of gagging (recorded as YES/NO)

- incidence of nausea and vomiting (recorded as YES/NO) 
- intensity of nausea, measured using verbal rating scale (VRS-11) from $0=$ no nausea at all to $10=$ maximal uncomfortable nausea

- use of midazolam as rescue medication

- use of propofol as rescue medication

- intensity of gagging, assessed by the practitioner, who will perform the TEE procedure using verbal rating scale (VRS-11) from $0=$ no gagging at all to $10=$ maximal gagging

- patients' satisfaction with relief of unwanted side effects during TEE procedure using verbal rating scale (VRS-5) from 1 = excellent to 5 = unsatisfactory

- patients' opinion to group allocation

- practitioners' (TEE examiner) opinion to group allocation

- heart rate and oxygen saturation (by the means of pulse oxymetry)

- side effects of acupuncture

\section{Sample size}

In order to calculate the appropriate sample size, we set the level of significance to 0.05 and power at $80 \%$. Based on the results of an investigation published by Rösler et al (9) and expecting to find a $30 \%$ difference in gagging intensity between the acupuncture group and the sham group (with standard deviation $50 \%$ from mean value) the number of patients was calculated to be 25 per group. Expecting the drop-out rate up to $15 \%$ in clinical trials, the number of patients per group was increased to 30 , in total 60 patients for the investigation. For sample size calculation the online software (free access) was used (13).

\section{Data analysis}

Normally distributed continuous data will be compared using the Student's t-test and an analysis of variance, as appropriate. Skewed data will be compared using Kruskall-Wallis and Friedman test. Chisquare test will be used to analyze the incidence of gagging and rescue midazolam application and success of patients' blinding. Statistical analysis will be performed using the software Statistical Package for Social Science, version 22.0 (SPSS, IBM Corporation, New York, USA).

\section{Discussion}

In this protocol, we describe a prospective randomized, patient, practitioner and assessor-blinded, singlecenter trial, which will compare real acupuncture with a non-penetrating sham procedure for treatment of gagging during transesophageal echocardiography (TEE). The aim of this clinical investigation is to develop an effective, easily applied method to relieve gag reflex using needling of acupoints CV24 and PC6 in patients scheduled for ambulatory TEE for cardiologic diagnostics. 
Gagging during TEE is extremely unpleasant for patients and may disturb the TEE-examiner, who is trying to perform a safe and effective diagnostic procedure (14). Premedication with midazolam was shown to improve patient comfort during and after TEE (15); however, extensive use of sedatives is associated with prolonged sedation, delayed recovery and need for prolonged observation prior to safe discharge for ambulatory patients (16). Acupuncture could be a safe and easy applied method to avoid pharmacological sedation in patients scheduled to ambulatory TEE procedures.

In this project we will use intradermal fixed needles for acupuncture during the TEE procedure for the first time. According to our experience, the practitioner, performing TEE, often comes into contact with long acupuncture needles, placed at acupoint CV24, which is situated in mentolabial groove (Figure 1). Such accidental contacts may cause a painful reaction, which could lead to uncontrolled withdrawal movement of patients during TEE examination. Thus we have decided to use intradermal needles, which will not disturb the TEE examination (manipulation with TEE probe in perioral area).

The combination of two acupoints CV24 and PC6 are used for the first time for this indication, and may allow relief of both gagging and nausea during TEE. The procedure will still be easy enough to apply in routine clinical practice when compared with previous protocols, where 10 various acupoints were successfully used to relieve stress and gagging in patients undergoing gastroscopy (17).

Although we have based our choice of control condition on experts' recommendations for optimal control procedures for clinical acupuncture trials (18), use of physiologically inactive sham control condition may introduce at least two sorts of bias into the present investigation: i) inactive sham lacks the non-specific physiological effect, that might indirectly enhance the measured specific effect of acupuncture, measured vs. inactive sham only (19); ii) probably some participants, who will randomly receive New Pyonex sham needles, will be able to distinguish them from real acupuncture, even despite the use of tip pressure from the neural pen, which imitates the pricking sensation. Nevertheless, we will carefully monitor this important aspect during our investigation, interviewing the patients and practitioners about their opinion regarding the group allocation.

In conclusion, we describe a proposal for a prospective randomized, patient, practitioner and assessorblinded, single-center trial with two arms/groups: real acupuncture will be compared to non-penetrating sham acupuncture.

\section{Trial Status}

Protocol version 2.0 (13.11.2018). The trial started January 2019 and is currently recruiting. The planned end of the study is the end of December 2019.

\section{Declarations}

MS: manuscript; STRICTA: Standards for Reporting Interventions in Clinical Trials of Acupuncture; TEE: transesophageal echocardiography 
- $\quad$ Acknowledgements

The authors thank Dr. Mike Cummings for re-checking the manuscript for mistakes and Dr. Ines Kiesow for her help in preparation of the figures.

Funding: This trial was conducted with no external funding and was instead funded from the institutional sources oft he Departments of Anesthesiology and Internal Medicine, University Medicine of Greifswald.

\section{Authors' contributions}

TU designed the study, calculated the sample size and currently performs the study interventions. IMK drafted the manuscript (MS) and enrolls the patients. SK drafted and revised the MS. KH drafted and revised the MS. RB enrolls the patients, revised and approved the MS. MB enrolls the patients, drafted and revised the MS. All authors have read and approved the final version of the MS.

\section{Availability of data and material}

Data will be available on request to the corresponding author

\section{Ethics approval and consent to participate}

The Ethics Commission of the University Medicine of Greifswald approved this study on October 30, 2018 (Reference number BB 166/18). Consent to participate/informed consent will be obtained from all participants in the study.

\section{Consent for publication}

Not applicable

\section{Competing interests}

The authors declare that they have no competing interests.

\section{References}

1. Davies AE, Kidd D, Stone SP, MacMahon J. Pharyngeal sensation and gag reflex in healthy subjects. Lancet 1995;345:487-8. 2. Hughes TAT, Wiles CM. Palatal and pharyngeal reflexes in health and motor neuron disease. J Neurol Neurosurg Psychiatry 1996;61:96-8. 3. Bassi GS, Humphris GM, Longman LP. 
The etiology and management of gagging: a review of the literature. J Prosthet Dent 2004;91(5):459-67. 4. Parker C, Alexandridis E, Plevris J, O'Hara J, Panter S. Transnasal endoscopy: no gagging no panic! Frontline Gastroenterol 2016;7(4):246-256. 5. Prashanti E, Sumanth KN, Renjith George P, Karanth L, Soe $\mathrm{HH}$. Management of gag reflex for patients undergoing dental treatment. Cochrane Database Syst Rev 2015;10:CD011116. 6. Fiske J, Dickinson C. The role of acupuncture in controlling the gagging reflex using a review of ten cases. Br Dent J 2001;190(11):611-3. 7. Rosted P, Bundgaard M, Fiske J, Pedersen $\mathrm{AM}$. The use of acupuncture in controlling the gag reflex in patients requiring an upper alginate impression: an audit. Br Dent J 2006;201(11):721-5 8. Mitchell J, Jeffrey S, Lochhead V. Use of acupuncture to reduce gagging during insertion of an oral airway. Anaesthesia 2008;63:1387-91. 9. Rösler A, Otto B, Schreiber-Dietrich D, Steinmetz H, Kessler KR. Single-needle acupuncture alleviates gag reflex during transesophageal Echocardiography: a blinded, randomized, controlled pilot trial. J Alternat Complement Med 2003;9:847-849. 10. Bilello G, Fregapane A. Gag reflex control through acupuncture: a case series. Acupunct Med 2014;32(1):24-7. 11. Goel H, Mathur S, Sandhu M, Jhingan P, Sachdev V. Effect of Low-level LASER Therapy on PC6 Acupoint to Control Gag Reflex in Children: A Clinical Trial. J Acupunct Meridian Stud 2017;10(5):317-323. 12. Usichenko TI, Lysenyuk VP, Groth MH, Pavlovic D. Detection of ear acupuncture points by measuring the electrical skin resistance in patients before, during and after orthopedic surgery performed under general anesthesia. Acupunct Electrother Res 2003;28(34):167-73. 13. https://www.stat.ubc.ca/ rollin/stats/ssize/n2.html (accessed September 29th 2018). 14. Chan KL, Cohen GI, Sochowski RA, Baird MG. Complications of transesophageal echocardiography in ambulatory adult patients: analysis of 1500 consecutive examinations. J Am Soc Echocardiogr 1991;4(6):577-82. 15. Aeschbacher BC, Portner M, Fluri M, Meier B, Lüscher TF. Midazolam premedication improves tolerance of transesophageal echocardiography. Am J Cardiol 1998;81(8):1022-6. 16. MauriceSzamburski A, Auquier P, Viarre-Oreal V, et al. Effect of sedative premedication on patient experience after general anesthesia: a randomized clinical trial. JAMA 2015;313:916-25. 17. Cahn AM, Carayon P, Hill C, Flammant R. Acupuncture in gastroscopy. Lancet 1978;28:182-183. 18. Lundeberg T, Lund I, Näslund J, Thomas M. The Emperors sham - wrong assumption that sham needling is sham. Acupunct Med 2008;26(4):239-42. 19. Vickers AJ. Placebo controls in randomized trials of acupuncture. Eval Health Prof 2002 Dec;25(4):421-35.

\section{Table}

Table 1 Interventions details by Standards for Reporting Interventions in Clinical Trials of Acupuncture (STRICTA) items 


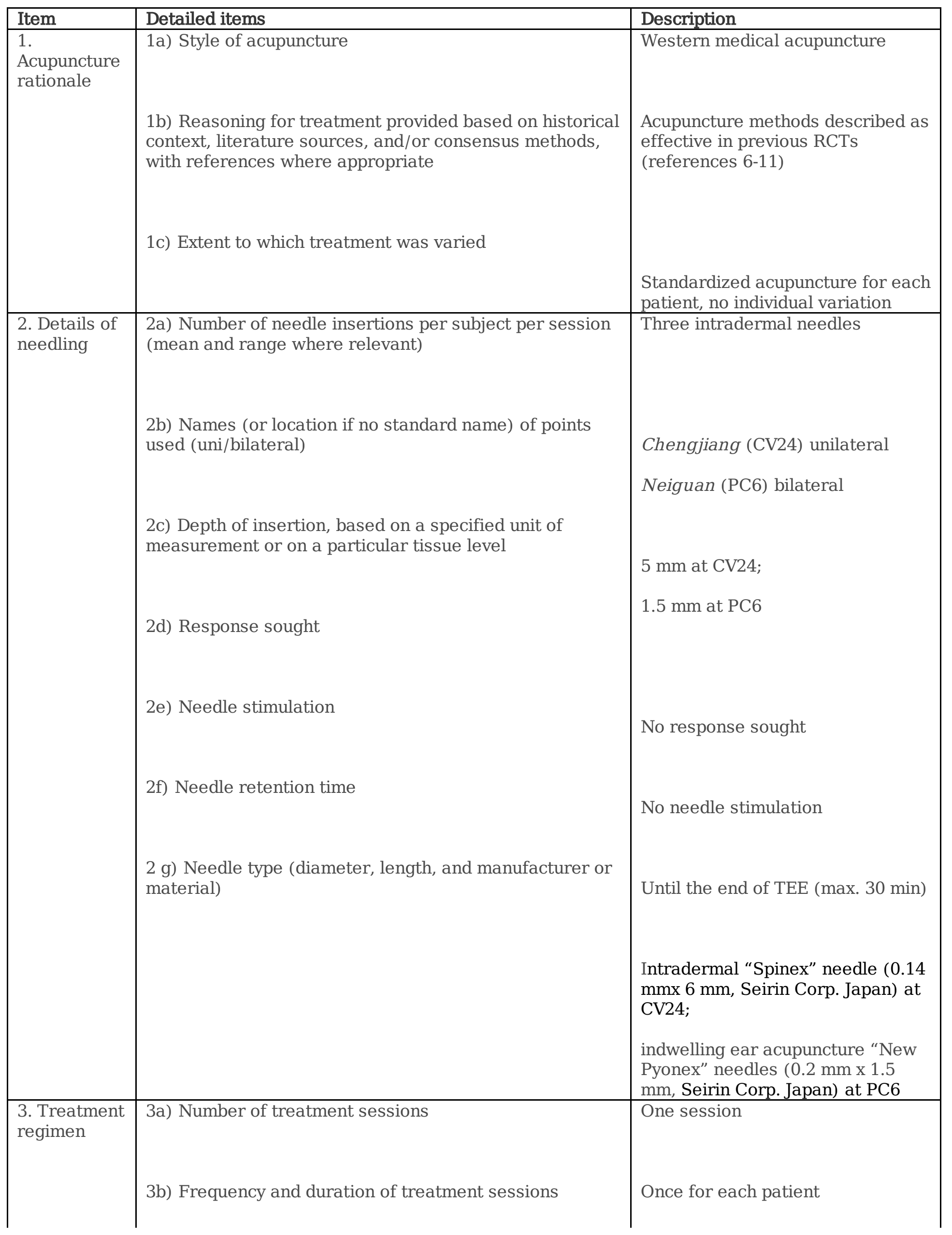

Page 10/16 


\begin{tabular}{|c|c|c|}
\hline \multirow{2}{*}{$\begin{array}{l}\text { 4. Other } \\
\text { components } \\
\text { of treatment }\end{array}$} & $\begin{array}{l}\text { 4a) Details of other interventions administered to the } \\
\text { acupuncture group }\end{array}$ & None \\
\hline & $\begin{array}{l}\text { 4b) Setting and context of treatment, including } \\
\text { instructions to practitioners, and information and } \\
\text { explanations to patients }\end{array}$ & $\begin{array}{l}\text { Each patient will be informed } \\
\text { about acupuncture or sham } \\
\text { procedure against gagging during } \\
\text { TEE }\end{array}$ \\
\hline $\begin{array}{l}\text { Practitioner } \\
\text { background }\end{array}$ & $\begin{array}{l}\text { 5) Description of participating acupuncturists } \\
\text { (qualification or professional affiliation, years in } \\
\text { acupuncture practice, other relevant experience) }\end{array}$ & $\begin{array}{l}\text { Licensed medical acupuncturist } \\
\text { with more than } 10 \text { years of } \\
\text { acupuncture practice }\end{array}$ \\
\hline \multirow[t]{2}{*}{$\begin{array}{l}\text { 6. Control of } \\
\text { comparator } \\
\text { interventions }\end{array}$} & $\begin{array}{l}\text { 6a) Rationale for the control or comparator in the } \\
\text { context of the research question, with sources that justify } \\
\text { this choice }\end{array}$ & $\begin{array}{l}\text { To study the efficacy and safety of } \\
\text { acupuncture in relief of gagging } \\
\text { during routine TEE procedure }\end{array}$ \\
\hline & $\begin{array}{l}\text { 6b) Precise description of the control or comparison } \\
\text { group. If sham acupuncture or any other type of } \\
\text { acupuncture-like control is used, provide details as for } \\
\text { items } 1 \text { to } 3 \text { above }\end{array}$ & $\begin{array}{l}\text { Sham needles will be placed over } \\
\text { the same sites in patients from the } \\
\text { control group as in acupuncture } \\
\text { group }\end{array}$ \\
\hline
\end{tabular}

TEE: transesophageal echocardiography

\section{Figures}


Figure 1.

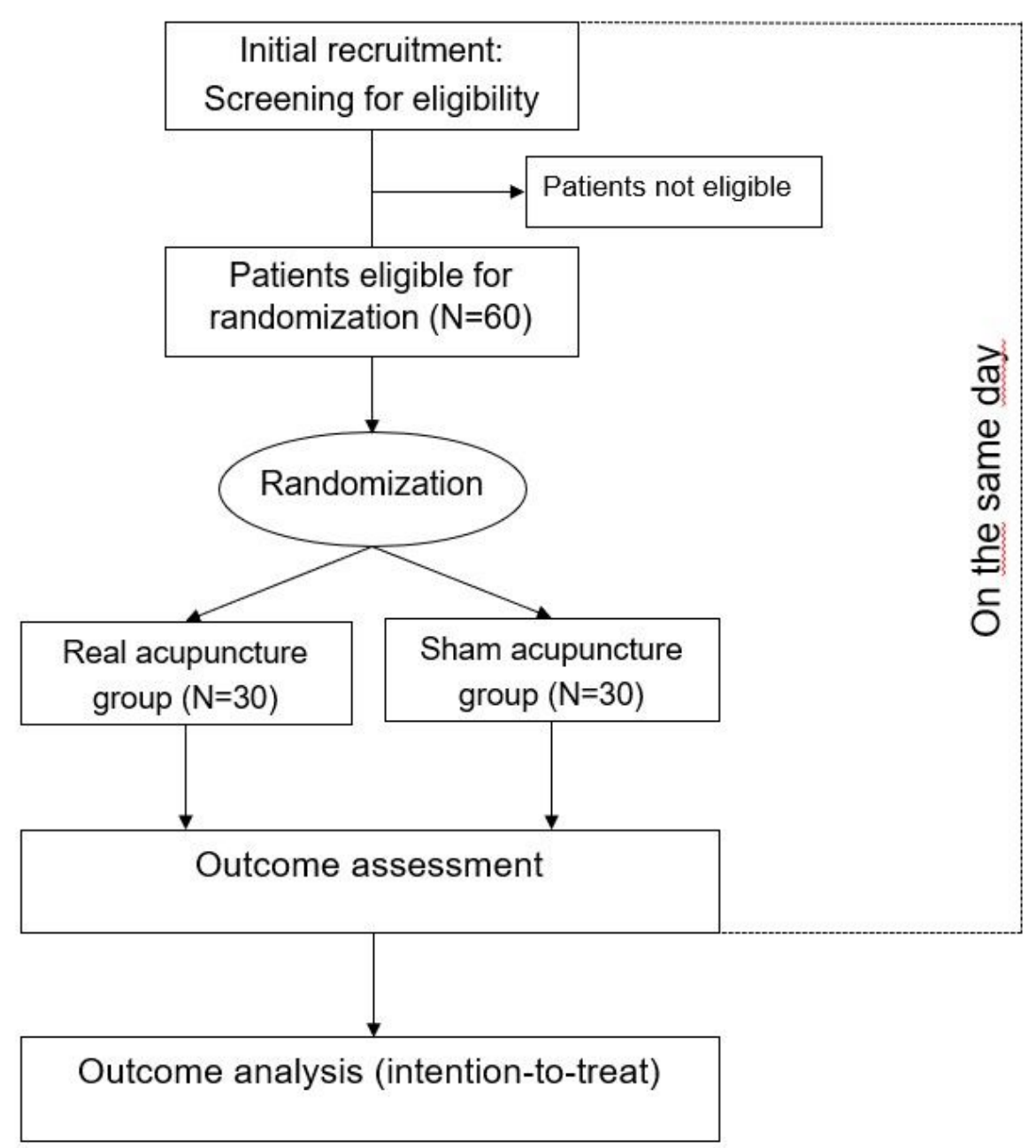

Figure 1

Flow of the investigation. 
Figure 2.

\begin{tabular}{|c|c|c|c|c|c|c|}
\hline \multirow[b]{3}{*}{ TIMEPOINT** } & \multicolumn{6}{|c|}{ STUDY PERIOD } \\
\hline & \multirow{2}{*}{$\begin{array}{c}\text { Enrolment } \\
T_{0-} \\
10 \min \\
\end{array}$} & \multirow{2}{*}{$\begin{array}{c}\text { Allocation } \\
T_{0}\end{array}$} & \multicolumn{3}{|c|}{ Post-allocation } & \multirow{2}{*}{$\begin{array}{c}\text { Close-ou } \\
T_{0}+ \\
20 \min \\
\end{array}$} \\
\hline & & & $\begin{array}{r}T_{0+} \\
5 \min \\
\end{array}$ & $\begin{array}{c}T_{0+}+ \\
10 \min \end{array}$ & $\begin{array}{c}T_{0}+ \\
15 \min \end{array}$ & \\
\hline \multicolumn{7}{|l|}{ ENROLMENT: } \\
\hline \multirow{3}{*}{$\begin{array}{c}\text { Informed consent } \\
\text { Randomization }\end{array}$} & $\mathrm{X}$ & & & & & \\
\hline & $\mathrm{X}$ & & & & & \\
\hline & & $\mathrm{X}$ & & & & \\
\hline Allocation & & $\mathrm{X}$ & & & & \\
\hline \multicolumn{7}{|l|}{ INTERVENTIONS: } \\
\hline \multicolumn{7}{|l|}{ Real acupuncture } \\
\hline \multicolumn{7}{|l|}{ Sham acupuncture } \\
\hline \multicolumn{7}{|l|}{ ASSESSMENTS: } \\
\hline $\begin{array}{l}\text { Incidence and } \\
\text { intensity of gagging }\end{array}$ & & & & & & $\mathrm{X}$ \\
\hline $\begin{array}{r}\text { Incidence and } \\
\text { intensity of nausea }\end{array}$ & & & & & & $\mathrm{X}$ \\
\hline $\begin{array}{r}\text { Use of rescue } \\
\text { medication }\end{array}$ & & & & & & $\mathrm{X}$ \\
\hline $\begin{array}{r}\text { Patients' satisfaction } \\
\text { with relief of } \\
\text { unwanted side effects }\end{array}$ & & & & & & $\mathrm{X}$ \\
\hline $\begin{array}{l}\text { Participants' opinion } \\
\text { to group allocation }\end{array}$ & & & & & & $\mathrm{X}$ \\
\hline \multicolumn{7}{|l|}{$\begin{array}{r}\text { Heart rate and } \\
\text { oxygen saturation }\end{array}$} \\
\hline $\begin{array}{r}\text { Side effects of } \\
\text { acupuncture }\end{array}$ & & & & & & $\mathrm{X}$ \\
\hline
\end{tabular}

Figure 2

Schedule of enrollment, intervention and outcome measures assessment. 
Figure 3.
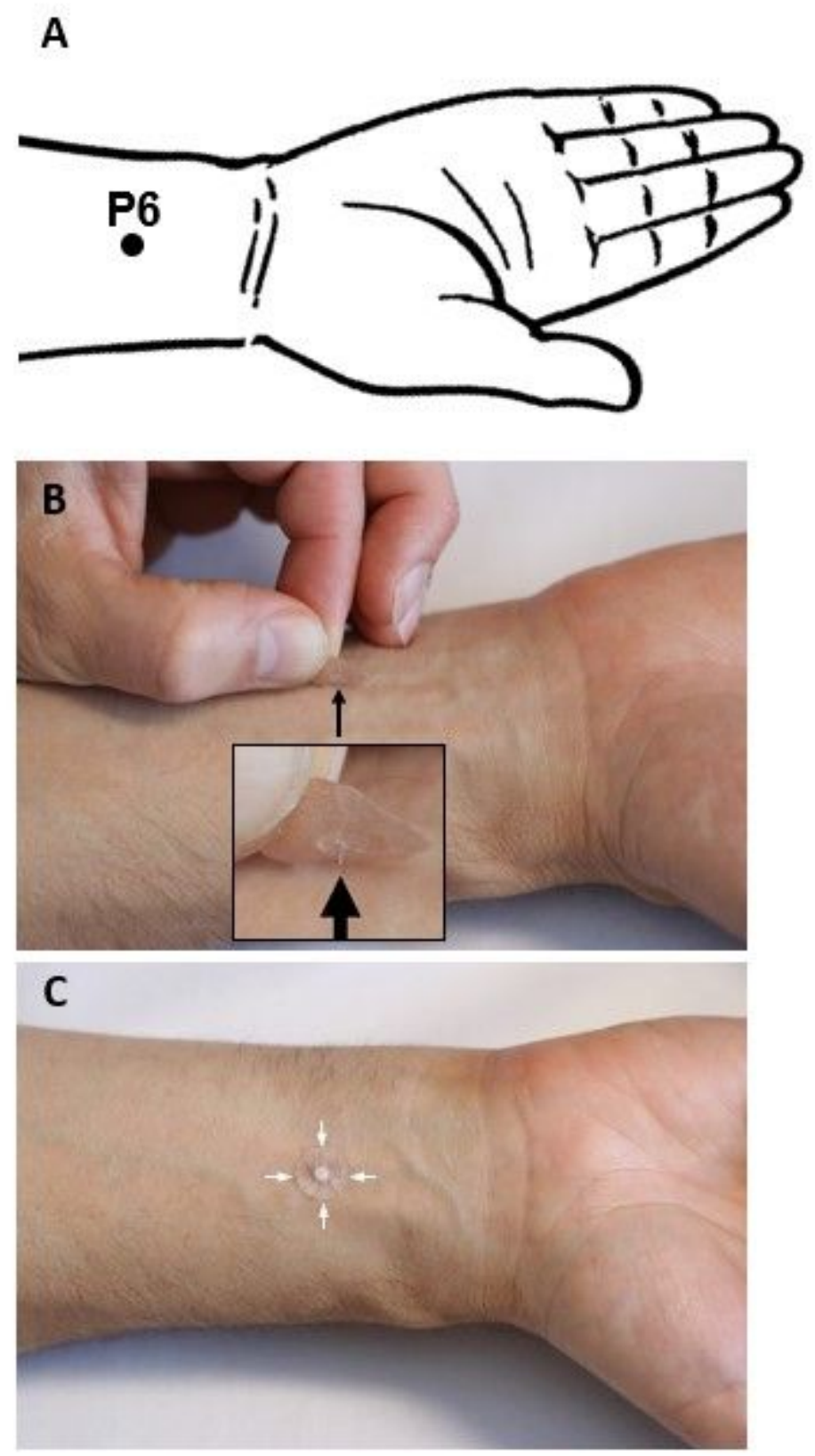

\section{Figure 3}

A: Acupoint Neuguan PC6 is situated on the ventral side of the forearm between the tendons of M. flexor carpi radialis and M. palmaris longus 3-4 $\mathrm{cm}$ proximal to the wrist crease; $\mathrm{B}$ : disposable indwelling acupuncture New Pyonex needle (black arrow) with diameter 0.2 and length $1.5 \mathrm{~mm}$, embedded in skincolored adhesive tape, over the acupoint PC6. C: New Pyonex needle in situ. White plastic hemisphere is seen over the round adhesive tape (white arrows). 
Figure 4.
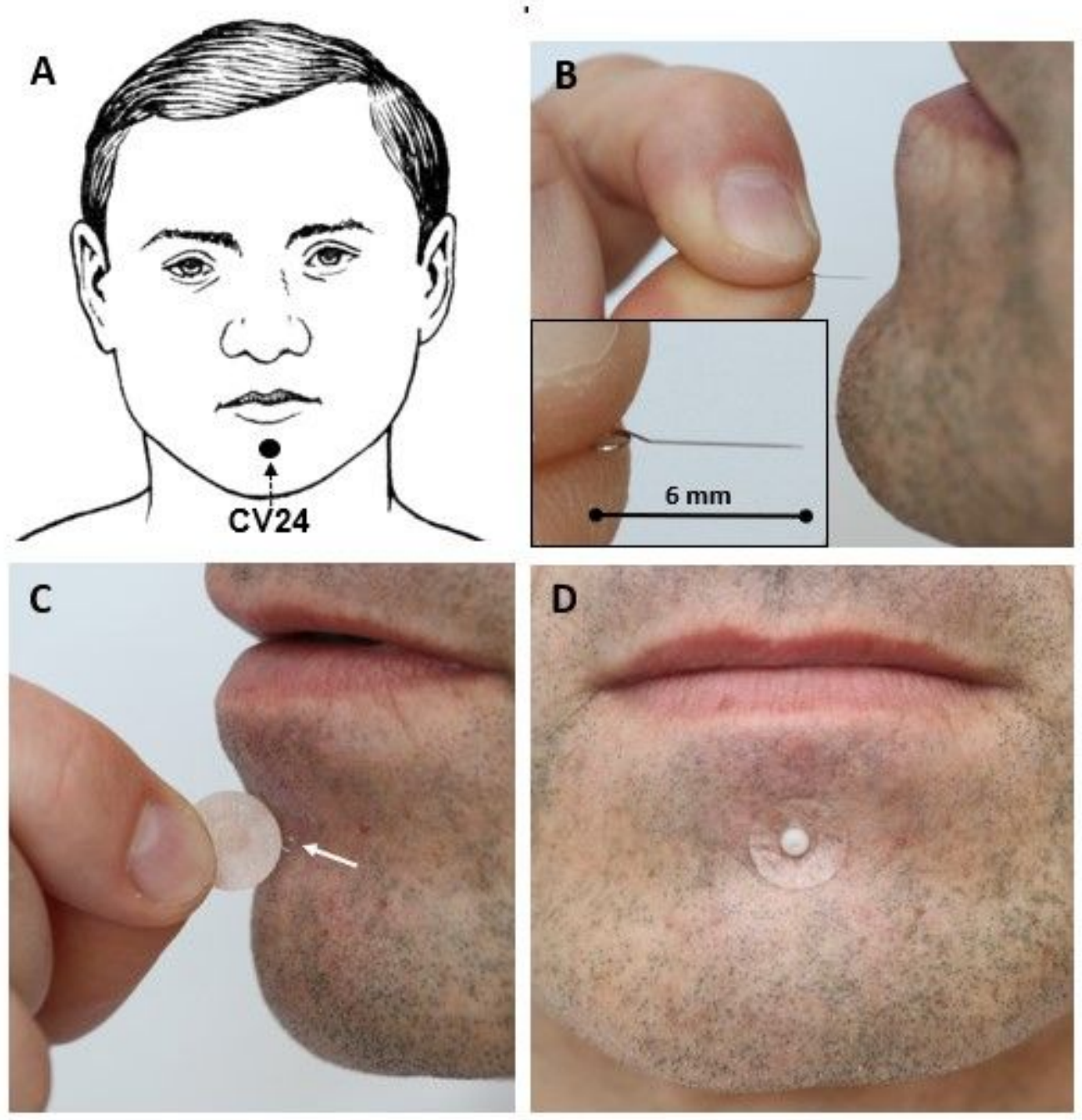

\section{Figure 4}

A: acupoint Chengjiang CV24 is situated on the ventral midline in the mento-labial groove. B: disposable intradermal "Spinex" needles with diameter $0.14 \mathrm{~mm}$ and length $6 \mathrm{~mm}$ over the CV24 acupoint. C: the loop of the inserted "Spinex" needle is seen on the surface of the skin (white arrow), New Pyonex sham device in the fingers. D: New Pyonex sham device is attached over "Spinex" needle.

\section{Supplementary Files}


This is a list of supplementary files associated with this preprint. Click to download.

- SPIRITChecklistMSUsichenkoetal.doc 